OPEN ACCESS

Edited by:

Ahmed Toosy,

University College London,

United Kingdom

Reviewed by:

Mark Paine

Royal Brisbane and Women's

Hospital, Australia

Christopher Charles Glisson,

Michigan State University,

United States

*Correspondence:

Shihui Wei

Shihuiwei706@163.com

Yong Tao

drtaoyong@sina.com

Specialty section

This article was submitted to

Neuro-Ophthalmology,

a section of the journal

Frontiers in Neurology

Received: 30 July 2020 Accepted: 05 October 2020

Published: 13 November 2020

Citation:

Kang H, Li H, Ai N, Liu H, Xu Q, Tao Y and Wei S (2020) Markedly Elevated

Serum Level of T-Helper Cell 17-Related Cytokines/Chemokines in

Acute Myelin Oligodendrocyte

Glycoprotein Antibody-Associated

Optic Neuritis.

Front. Neurol. 11:589288.

doi: 10.3389/fneur.2020.589288

\section{Markedly Elevated Serum Level of T-Helper Cell 17-Related Cytokines/Chemokines in Acute Myelin Oligodendrocyte Glycoprotein Antibody-Associated Optic Neuritis}

\author{
Hao Kang ${ }^{1}$, Hongyang $\mathrm{Li}^{2}$, Nanping $\mathrm{Ai}^{3}$, Hongjuan $\mathrm{Liu}^{3}$, Quangang $\mathrm{X} \mathrm{u}^{3}$, Yong Tao ${ }^{1 *}$ and \\ Shihui Wei ${ }^{3 *}$
}

\footnotetext{
${ }^{1}$ Department of Ophthalmology, Beijing Chaoyang Hospital, Capital Medical University, Beijing, China, ${ }^{2}$ Department of Ophthalmology, Beijing Friendship Hospital, Capital Medical University, Beijing, China, ${ }^{3}$ Department of Ophthalmology, The Chinese People's Liberation Army General Hospital, Beijing, China
}

Purpose: The purpose of this study was to examine the differences in immunopathogenesis based on the cytokine/chemokine profiles in myelin oligodendrocyte glycoprotein antibody (MOG-lgG)-positive and -negative groups.

Methods: We measured the levels of T-helper cell 17 (Th17) cell-related cytokines/chemokines in 74 serum samples, which were divided into four groups: healthy control $(H C)$ group $(n=15)$, idiopathic demyelinating optic neuritis (IDON) group $(n=20)$, aquaporin 4 (AQP4)-lgG-positive optic neuritis (ON) group $(n=18)$, and MOG-lgG positive-ON group $(n=21)$. Serum IL17, IL21, IL28, IL31, CXCL1, CXCL2, CCL2, CCL11, CCL20, and LT- $\alpha$ were detected.

Results: The serum of the MOG-IgG-positive ON patients showed an obvious elevation of Th17 cell-related cytokines/chemokines compared with that of all the MOG-IgG-negative ON patients. Serum IL17 and IL21 were significantly higher in the ON patients with MOG-IgG positive than in all the other three groups. The serum levels of IL28, IL31, CXCL1, and CCL11 were higher in the ON patients with MOG-IgG positive than in the HC group and the IDON group. The serum concentration of CCL2, CXCL2, and CCL20 in the MOG-IgG-positive and AQP4-lgG-positive group is higher than that of the HC group. No difference in serum LT- $\alpha$ level was found among the four groups. Adjusted multiple regression analyses showed a positive association of IL17 and IL21 levels with the serum concentration of MOG-IgG in the ON patients.

Conclusion: The elevated serum level of Th17 cell-related cytokine/chemokines may play an important role in the pathogenesis of MOG-lgG-positive demyelinating ON.

Keywords: T helper cell 17 (Th17), cytokines, chemokines, optic neuritis, MOG-IgG, AQP4-IgG 


\section{INTRODUCTION}

Optic neuritis (ON) is the most common optic neuropathy affecting young adults. ON can occur in isolation, or as the initial symptom of autoimmune-mediated demyelinating diseases, such as multiple sclerosis (MS) or neuromyelitis optica (NMO)/neuromyelitis optica spectrum disorders (NMOSD) (1). In most cases, $\mathrm{NMO}$ is caused by autoantibodies to aquaporin 4 (AQP4-IgG) $(2,3)$, but $10-20 \%$ of patients with NMO are negative for AQP4-IgG $(4,5)$. Recent studies have shown the presence of IgG antibodies to myelin oligodendrocyte glycoprotein antibody (MOG-IgG) in some NMO/NMOSD patients $(6,7)$. MOG-IgG is pathogenic in human demyelinating diseases, and it is a biomarker of autoimmune $\mathrm{ON}$ and longitudinally extensive transverse myelitis (LETM) $(8,9)$.

MOG-IgG-seropositive patients had some clinical features different from those with AQP4-IgG seropositive (7). In addition, the histopathology of brain and spinal cord lesions of MOG IgG+ patients has been demonstrated to be different from that of AQP4-IgG+ patients $(10,11)$. MOG-IgG-related disease is now considered as a disease entity in its own right, immunopathogenetically distinct from MS and from AQP4-IgGrelated demyelinating diseases.

Cytokines/chemokines are biologically active intercellular messengers having pleiotropic effects on various cell types resulting in immune system activation (12) In the nervous system, cytokines, and chemokines are involved in the regulation of central nerve system (CNS)-immune system interactions that function as neuromodulators and control neurodevelopment, neuroinflammation, and synaptic transmission (13). CD4+ T helper cells can be divided into four major subsets, and Th17 lineage is a recently discovered subset of CD4+ T-helper cells, which can promote tissue inflammation by induction of inflammatory mediators and recruitment of inflammatory cells (14). Th17 cells coordinate local tissue inflammation through the regulation of inflammatory cytokines and chemokines such as IL-17, IL-21, IL-28, CXCL1, CCL2, CXCL2, CCL11, and CCL20 (15). In this study, we evaluated the levels of Th17-related cytokines and chemokines in serum samples from MOG-IgGseropositive ON, AQP4-IgG-seropositive ON, and idiopathic demyelinating ON (IDON) patients in the acute phase, in order to investigate the differences in immunopathogenesis based on the cytokine/chemokine profiles.

\section{MATERIALS AND METHODS}

\section{Patients}

In this study, 59 patients with unilateral or bilateral isolated ON were recruited from the Ophthalmology Department of Beijing Chaoyang Hospital of Capital Medical University and the Chinese People's Liberation Army General Hospital (PLAGH). Recruitment was completed between April 2017 and July 2018. ON was the first symptom in all the patients who fulfilled the diagnosis criteria of ON. All the subjects were treated with methylprednisolone according to the suggestion with ONTT (16). If a minimal response to the corticosteroid therapy and the vision remained below 0.1 were clinically observed, the patient involved was given a total of three to five plasma exchanges.

All blood samples were collected during the acute phase of the disease or within a month of exacerbation. Seventy-four serum samples were drawn from the patients with acute demyelinating $\mathrm{ON}$ and from the controls, including 15 samples from the healthy control (HC) group, 20 samples from the IDON group, 18 samples from the AQP4-IgG-seropositive ON group, and 21 samples from the MOG-IgG-seropositive ON group.

Ophthalmic examinations including best-corrected visual acuity (BCVA), intraocular pressure, slit lamp examination, pupillary reactions in unilateral or bilateral asymmetric conditions, and ocular fundus examinations were conducted by professional ophthalmologists.

BCVA was tested by using a Snellen chart and was transformed into logarithm of the minimum angel of resolution (logMAR) values by using Petzold's et al. (17) VA conversion method. If a VA was below 0.01, finger-counting (FC), hand motion (HM), perception of light (LP), and no perception of light (NLP) were tested, and the results were documented accordingly. All patients underwent visual field, electrodiagnostic tests, and orbit and brain magnetic resonance imaging (MRI) examination.

\section{Aquaporin $4 \mathrm{lgG}$ and Myelin Oligodendrocyte Glycoprotein Antibody Testing}

All serum samples were analyzed for the presence of AQP4IgG by an extracellular live cell-staining immunofluorescence technique using transiently transfected AQP4-expressing cells as previously described (18). Samples were scored as positive or negative by at least two independent experiments. A dilution of 1:1,000 was employed as the maximum positive value and 1:10 as the cut-off for positive and negative cases. MOGIgG detection was performed by CBA with full-length human MOG-transfected HEK293 cells. MOG-IgG titers of $\geq 1: 10$ were classified as positive.

\section{Cytokine and Chemokine Assay}

Th17-related cytokines (IL-17, IL-21, IL-28, and IL-31) and chemokines (CXCL1/GRO alpha, CXCL2/GRO beta, CCL2/MCP-1, CCL20/MIP-3, and CCL11/eotaxin) and LTalpha/TNF-beta were measured by means of ELISA. The regression equation of the standard curve $\left(R^{2}>0.98\right)$ was calculated on the basis of the standard concentration and the corresponding $A$-value. Similarly, the corresponding sample concentration was calculated with reference to the sample's $A$-value.

\section{Ethics Statement}

This study was approved by the Ethics Committee of Beijing Chaoyang Hospital and PLAGH, and was conducted following the Declaration of Helsinki in its currently applicable version. Written informed consents were obtained from each patient.

\section{Statistical Analysis}

Statistical analysis was performed by using SPSS for Windows, Version 21.0. Continuous variables were analyzed using a 
TABLE 1 | Epidemiologic and disease characteristics of ON patients and healthy controls.

\begin{tabular}{|c|c|c|c|c|c|}
\hline & IDON & $\mathrm{AQP} 4+\mathrm{ON}$ & $M O G+O N$ & $\mathrm{HC}$ & $P$-value \\
\hline Number of patients & 20 & 18 & 21 & 15 & - \\
\hline Age at onset (years) & $34.50 \pm 12.96$ & $37.06 \pm 12.95$ & $35.81 \pm 14.08$ & $35.20 \pm 12.39$ & 0.945 \\
\hline Gender (male:female) & $3: 17$ & $1: 17$ & $11: 10$ & $4: 11$ & 0.005 \\
\hline Ocular pain $(n, \%)$ & $10(50.0)$ & 15 (83.3) & $17(81.0)$ & - & 0.036 \\
\hline Disc swelling ( $n, \%)$ & $8(40.0)$ & 7 (38.9) & 7 (33.3) & - & 0.894 \\
\hline Bilateral, ever (\%) & $5(25.0)$ & $7(38.9)$ & $9(42.9)$ & - & 0.461 \\
\hline Recurrent ON onset $(n, \%)$ & $8(40.0)$ & $18(100.0)$ & $21(100.0)$ & - & $<0.001$ \\
\hline Median CSF white cell count (no. $/ \mathrm{mm}^{3}$ ) & $2.10 \pm 4.12$ & $6.89 \pm 11.29$ & $3.00 \pm 3.67$ & - & 0.095 \\
\hline CSF protein (mg/L) & $288.82 \pm 85.01$ & $422.53 \pm 132.88$ & $304.42 \pm 94.03$ & - & $<0.001$ \\
\hline CSF IgG level (mg/L) & $2.48 \pm 0.96$ & $3.55 \pm 3.02$ & $2.14 \pm 0.64$ & - & 0.05 \\
\hline BCVA at first ON attacks in acute time (logMAR) & $1.85 \pm 0.89$ & $2.17 \pm 0.79$ & $1.82 \pm 0.81$ & - & 0.369 \\
\hline BCVA recovery at last follow-up (logMAR) & $0.64 \pm 0.67$ & $1.00 \pm 0.78$ & $0.37 \pm 0.51$ & - & 0.015 \\
\hline
\end{tabular}

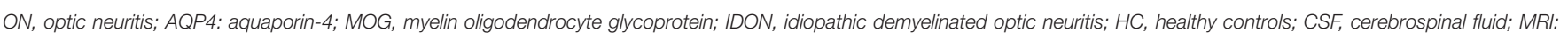
magnetic resonance imaging; BCVA, best-corrected visual acuity. The bold values are used to indicate values with $P<0.05$.

nonparametric test (Mann-Whitney U test). The Chi-squared test, or Fisher's exact test if applicable, was used to analyze the categorical data. The differences among any three groups were identified by using the ANOVA or Kruskal-Wallis test. In order to reduce type I errors, Bonferroni correction had been applied on the $P$-values. Correlation ranks were evaluated by Spearman's rank correlation tests.

\section{RESULTS}

\section{Demographic Data and Clinical Characteristics}

The demographic data and clinical characteristics of all the 59 Chinese ON patients were compared (Table 1). The mean age at disease onset was similar in the three groups. Female predominance was apparent in the IDON patients $(82.4 \%)$ and the AQP4-IgG seropositive-ON patient (94.1\%), but the MOG-IgG-seropositive ON patients were mostly male, with a male-to-female ratio of 11:10. Compared to the IDON group (10/20, 50.0\%), the AQP4-IgG-seropositive ON (15/18, 83.3\%) and MOG-IgG-seropositive ON (17/21, 81.0\%) patients were more likely to have accompanying ocular pain. The proportion of disc swelling and monocular/binocular involvement showed no difference between the three groups. Recurrent $\mathrm{ON}$ appeared frequently among all the AQP4-IgG-seropositive ON $(18 / 18,100.0 \%)$ and MOG-IgG-seropositive ON patients $(21 / 21$, $100.0 \%)$, and the IDON patients $(8 / 20,60 \%)$ had a more frequent monophasic course. In the routine CSF analysis, no significant difference in the median CSF white cell count $\left(\mathrm{no} / \mathrm{mm}^{3}\right)$ and the CSF IgG level (mg/L) between the three groups was found. CSF total protein $(\mathrm{mg} / \mathrm{L})$ was significantly higher in the AQP4IgG-seropositive ON group than that in the other two groups $(P<0.001$; AQP4-IgG $+\mathrm{ON}$ vs. MOG-IgG $+\mathrm{ON}: P=$ $0.003, \mathrm{AQP} 4-\mathrm{IgG}+\mathrm{ON}$ vs. IDON: $P=0.001)$. There were no significant differences between the three groups in the proportion of optic lesion in MRI. None of the patients in the three groups had MRI results that met the radiological diagnostic criteria of
MS or NMO. VA at first attacks in the acute phase and VA recovery at the last follow-up were compared in the AQP4IgG-seropositive ON, MOG-IgG-seropositive ON, and IDON patients. No differences in visual loss during the acute stage were observed between the three groups. At the last follow-up, the AQP4-IgG-seropositive ON patients were significantly more likely to get poor VA recovery over time than the other patients $(P=0.015$; AQP4-IgG + ON vs. MOG-IgG $+\mathrm{ON}: P=0.012$, AQP4-IgG + ON vs. IDON: $P=0.272$ ).

\section{Comparison of T-Helper Cell 17-Related Serum Cytokine/Chemokine Levels Between the Myelin Oligodendrocyte Glycoprotein Antibody-Seropositive Optic Neuritis, Aquaporin 4-IgG-Seropositive Optic Neuritis, Idiopathic Demyelinating Optic Neuritis Patients, and the Healthy Controls}

The dot plots of individual serum cytokines/chemokines levels are shown in Figure 1, and the values are summarized in Table 2. IL-17, IL-21, IL-28, IL-31, CXCL1, CXCL2, CCL2, CCL20, and CCL11 were significantly elevated in the MOGIgG-seropositive ON patients than in the MOG-IgG-patients. The mean concentration of IL-17 in the patients with MOGIgG-seropositive $\mathrm{ON}$ was much higher than the other three groups of patients. Moreover, it was also higher in the patients with AQP4-IgG-seropositive ON than in the HC group (IL-17: AQP4-IgG + ON vs. HC: $P=0.031)$. The concentration of IL-21 in the patients with MOG-IgG-seropositive ON was also higher than in the AQP4-IgG-seropositive ON, IDON, and HC groups. The IL-23 concentration in the patients with MOGIgG-seropositive ON was also higher than in the IDON and HC groups. The MOG-IgG-seropositive ON patients showed a significantly higher IL-31 level than the IDON and HC patients. The serum CXCL1 and CXCL2 concentration in the patients with MOG-IgG-seropositive ON and AQP4-IgG-seropositive ON was 

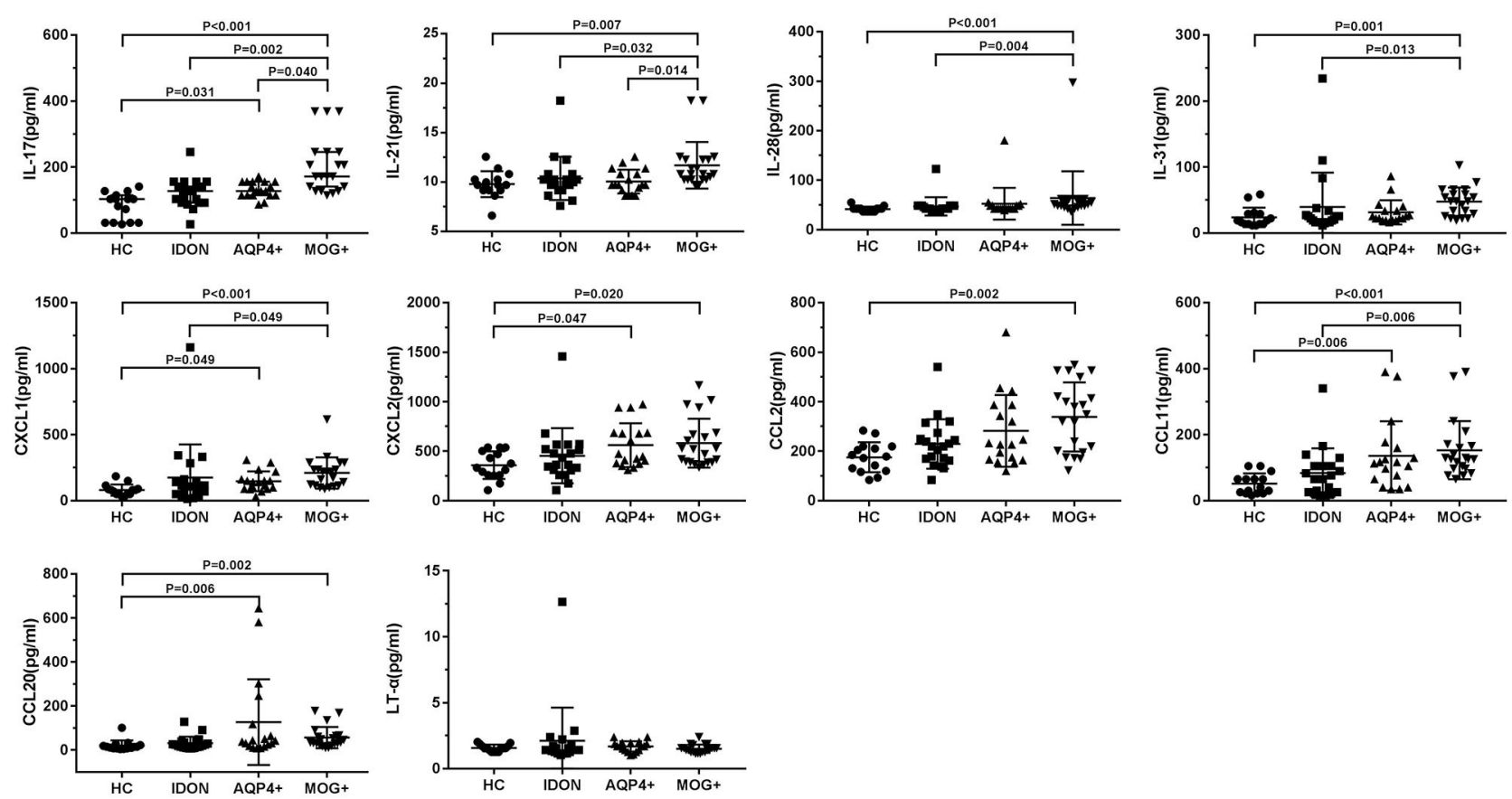

FIGURE 1 | Comparison of T-helper cell 17 (Th17)-related serum cytokine/chemokine levels between myelin oligodendrocyte glycoprotein antibody (MOG-IgG) + optic neuritis (ON), aquaporin 4 (AQP4-IgG) + ON, idiopathic demyelinating optic neuritis (IDON), and healthy controls.

TABLE 2 | Serum level of Th17-related cytokine/chemokines of optic neuritis patients.

\begin{tabular}{|c|c|c|c|c|c|c|c|c|}
\hline & MOG-IgG + ON $(n=21)$ & AQP4-IgG + ON $(n=18)$ & IDON $(n=20)$ & HC $(n=15)$ & $\mathbf{P}^{t}$ & $\mathbf{P} 1^{\S}$ & $\mathbf{P} 2^{\S}$ & $\mathbf{P 3}^{\S}$ \\
\hline IL-17 (pg/ml) & $231.58 \pm 156.74$ & $131.33 \pm 24.02$ & $122.07 \pm 44.56$ & $81.73 \pm 41.19$ & $<0.001$ & 0.040 & 0.002 & $<0.001$ \\
\hline IL-21 (pg/ml) & $11.70 \pm 2.35$ & $10.05 \pm 1.20$ & $10.38 \pm 2.19$ & $9.79 \pm 1.32$ & 0.002 & 0.014 & 0.032 & 0.007 \\
\hline IL-28 (pg/ml) & $63.99 \pm 54.00$ & $52.49 \pm 32.16$ & $46.98 \pm 18.46$ & $41.34 \pm 5.23$ & $<0.001$ & 0.081 & 0.004 & $<0.001$ \\
\hline IL-31 (pg/ml) & $47.51 \pm 21.48$ & $31.27 \pm 18.28$ & $39.61 \pm 51.85$ & $23.79 \pm 14.67$ & 0.001 & 0.167 & 0.013 & 0.001 \\
\hline CXCL1 (pg/ml) & $209.71 \pm 119.21$ & $146.49 \pm 74.65$ & $175.12 \pm 251.10$ & $80.06 \pm 42.96$ & $<0.001$ & 0.652 & 0.049 & $<0.001$ \\
\hline CCL20 (pg/ml) & $2.31 \pm 0.12$ & $1.17 \pm 0.21$ & $1.66 \pm 0.13$ & $19.84 \pm 23.87$ & 0.001 & $>0.99$ & 0.131 & 0.002 \\
\hline CCL11 (pg/ml) & $152.83 \pm 88.16$ & $135.75 \pm 104.86$ & $83.41 \pm 75.38$ & $21.34 \pm 31.11$ & $<0.001$ & $>0.99$ & 0.006 & $<0.001$ \\
\hline LT- $\alpha$ (pg/ml) & $1.51 \pm 0.31$ & $1.68 \pm 0.39$ & $2.11 \pm 2.52$ & $1.57 \pm 0.25$ & 0.42 & - & - & - \\
\hline
\end{tabular}

Th17, T-helper cell 17; ON, optic neuritis; AQP4, aquaporin 4; MOG, myelin oligodendrocyte glycoprotein; IDON, idiopathic demyelinated optic neuritis; HC, healthy controls; P1, MOGIgG + ON and AQP4-IgG + ON; P2, MOG-IgG + ON and IDON; P3, MOG-IgG + ON and HC; ${ }^{\dagger}$ Kruskal-Wallis H-test; §Bonferroni method. The bold values are used to indicate values with $P<0.05$.

also higher than that in the HC group (CXCL2: AQP4-IgG+ ON vs. HC: $P=0.047)$. The MOG-IgG-seropositive $\mathrm{ON}$ patients had a significantly higher CCL2 level than the HC patients. In the serum concentration of CCL20, the MOG-IgG-seropositive ON and AQP4-IgG-seropositive ON patients showed higher levels than the HC group (CCL20: AQP4-IgG + ON vs. HC: $P=0.006)$. CCL11 was significantly elevated in the two autoantibodyassociated ON patients than in the HC group (CCL11: AQP4IgG $+\mathrm{ON}$ vs. HC: $P=0.006)$. The CCL11 concentration in the MOG-IgG-seropositive ON patients was also higher than that in the IDON group. No significant difference was found in the serum concentration of LT- $\alpha$ between the four groups.

\section{Relationship Between T-Helper Cell} 17-Related Serum Cytokines/Chemokines and Serum Myelin Oligodendrocyte Glycoprotein Antibody Titer in the Myelin Oligodendrocyte Glycoprotein Antibody-Seropositive Optic Neuritic

\section{Patients}

We then analyzed the potential correlations between the elevated Th17-related cytokines/chemokines levels and the titer of MOG-IgG in 21 MOG-IgG-seropositive ON patients (Table 3). Correlation analyses showed that serum IL-17 was positively 
correlated with the titer of MOG-IgG in the patients' serum $(r=0.534, P=0.013$; Figure 2$)$. The serum concentration of CCL11 was negatively correlated with the titer of MOGIgG in the MOG-IgG-seropositive ON patients $(r=-0.481$, $P=0.027$; Figure 2). However, no significant correlation between the other cytokines/chemokines and the serum titer of MOG-IgG was observed.

\section{DISCUSSION}

In the present study, we compared the Th17-related serum cytokines/chemokines in healthy adults and ON patients with different etiologies. In the MOG-IgG-seropositive ON patients, we noted a significant upregulation of Th17 cell-related serum cytokines/chemokines. These data are in agreement with previous studies of Th17 changes in other autoimmune diseases, as Th17 cells are a key stakeholder in the pathogenesis of many autoimmune disorders (15).

TABLE 3 | Spearman's correlation coefficient ( $r$ ) of the association between serum cytokines/chemokines and MOG-IgG titer in 21 MOG-IgG-seropositive ON patients.

\begin{tabular}{|c|c|c|c|}
\hline & MOG IgG $(n=21)$ & Correlation coefficient & $P$ \\
\hline IL-17 (pg/ml) & & 0.534 & $0.013^{*}$ \\
\hline IL-21 (pg/ml) & & 0.163 & 0.479 \\
\hline IL-28 (pg/ml) & & -0.104 & 0.653 \\
\hline IL-31 (pg/ml) & & -0.195 & 0.397 \\
\hline CXCL1 (pg/ml) & & -0.218 & 0.344 \\
\hline CXCL2 (pg/ml) & & 0.280 & 0.220 \\
\hline CCL2 (pg/ml) & & -0.074 & 0.751 \\
\hline CCL20 (pg/ml) & & 0.176 & 0.445 \\
\hline CCL11 (pg/ml) & & -0.481 & $0.027^{\star}$ \\
\hline LT- $\alpha(\mathrm{pg} / \mathrm{ml})$ & & -0.187 & 0.416 \\
\hline
\end{tabular}

ON, optic neuritis; MOG, myelin oligodendrocyte glycoprotein. The bold values are used to indicate values with $P<0.05$.

${ }^{*} P<0.05$
Th17 cells have been involved in several autoimmune disorders, and they seem to be relevant in CNS autoimmunity development. Th17-related molecules have also been shown to correlate with parameters of disease activity and severity in CNS inflammatory demyelinating diseases (19). IL-17 is a proinflammatory cytokine that upregulates the expression of inflammatory genes. More importantly, elevated IL-17 levels have been observed in autoimmune diseases like MS, inflammatory bowel disease, psoriasis, and rheumatoid arthritis (20). Although IL-17 is the signature cytokine of Th17 cells, many studies have shown that other cytokines related with Th17 cells are also significant in the pathogenesis of inflammatory responses. The release of IL- 6 and IL- 21 by polyclonally activated CD4+ T cells obtained from NMO patients was shown to have direct correlations with neurological disability (14). IL-31 is also produced mainly by CD4 $+\mathrm{T}$ cells. A recent report has shown that the serum concentration of IL-31 significantly increased in NMOSD patients and positively correlated with the serum level of IL-17 in those patients (21). Moreover, Th17 cells necessitate a large quantity of chemokines and chemokine receptors to cross the blood-brain barrier (BBB), which enables them to disrupt the $\mathrm{BBB}$ and access the CNS through some different pathways. IL-17 is a key factor in the disruption of the BBB by directly impairing its integrity (22). In vitro and in vivo studies have shown that through the action of IL-17, Th17 cells can efficiently break down BBB tight junctions, bring out high levels of the cytolytic enzyme granzyme $\mathrm{B}$, and provide impetus to the recruitment of additional CD4+ lymphocytes from the systemic circulation into the CNS (23). In addition, Th17 cells are also capable of inducing CXCL1 and CXCL2, chemokines that are powerful attractants for polymorphonuclear cells, and of contributing much to the disruption of the BBB in experimental autoimmune encephalomyelitis (EAE) (24).

NMOSD is a severe CNS autoimmune inflammatory disorder, which has always been recognized as a B-cell-mediated humoral immune disease. However, B-cell depletion therapy was not efficacious in some NMOSD patients, including both antibodyseropositive or -seronegative patients (25). One possible reason is that B cell-mediated immunity may not be the sole contributor to NMOSD-like lesions, and other components like CD4+
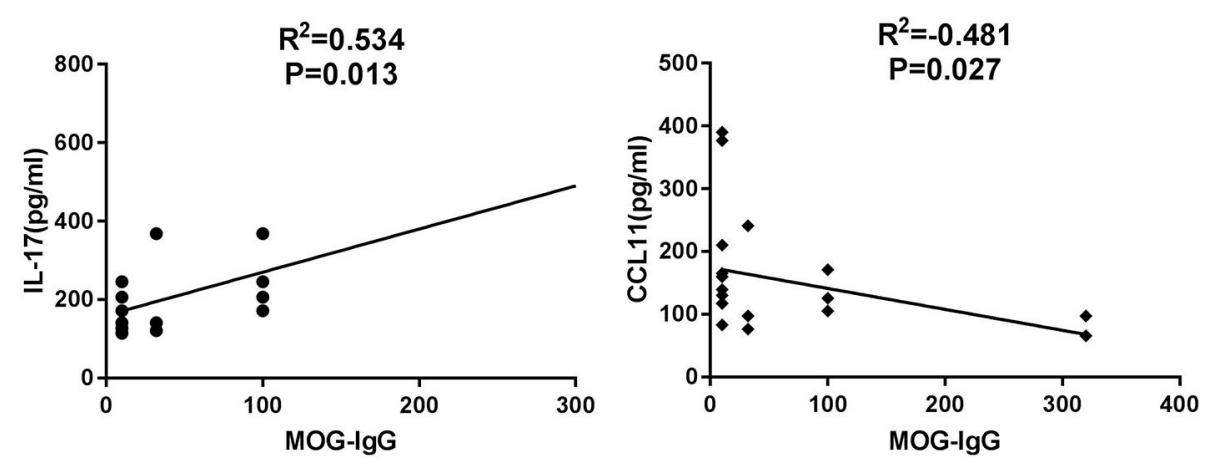

FIGURE 2 | Relationship between Th17-related serum cytokines/chemokines and serum MOG-lgG titer in MOG-IgG-seropositive ON patients. 
T cells, especially Th17 cells, may also play potential roles. Studies have demonstrated that T-cell-mediated immunity may participate in the pathological process of NMOSD, especially in the Th17 phenotype (26-28). Another possible explanation is that MOG-IgG-related demyelination in the optic nerve and the spinal cord can partly explain AQP4-IgG-seronegative NMOSD patients. In this study, the results showed that both AQP4-IgG-seropositive ON and MOG-IgG-seropositive ON have increased the serum concentration of Th17-related cytokines and chemokines compared with that in the control subjects, while the change in MOG-IgG-seropositive $\mathrm{ON}$ is more significant. This result supported the hypothesis that Th17 cells are highly activated in MOG-IgG-seropositive ON patients during the disease's acute exacerbation and relapse stages. From a clinical point of view, the difference in cytokines and chemokines between different types of ON suggests its value in the differential diagnosis of disease, especially in the early stage of diseases.

MOG-IgG has been detected in a proportion of AQP4-IgGseronegative NMOSD patients. MOG-IgG is a biomarker for patients with CNS demyelinating diseases that have distinct demographic, serologic, clinical, and radiologic features from classical MS and from AQP4-IgG-mediated NMOSD, suggesting that MOG-IgG might mediate a distinct disease (29). Moreover, the histopathology of the lesions of MOG-IgG-seropositive patients has been shown to differ from that of AQP4-IgGmediated CNS lesions $(10,11)$. In a retrospective multicenter study of MOG-IgG-seropositive NMOSD patients, $88 \%$ of the patients developed acute $\mathrm{ON}$ at least once, $56 \%$ of the patients developed acute myelitis at least once, $44 \%$ of the patients only had a history of ON but not of myelitis, while only $12 \%$ of the patients had a history of myelitis but not of ON (29). This is consistent with another study that demonstrated that the optic nerve is more susceptible than the spinal cord in MOG-IgG-related CNS autoimmunity (30). A recent study has shown that autoimmune inflammatory infiltrates in the optic nerve are different from inflammation in other parts of the CNS, suggesting that the optic nerve might be an immunologic compartment different from the spinal cord. Compared with the spinal cord, Th17 cells prevailed in the optic nerve and the brain. Local tissue expression of IL-17 was the highest in the optic nerve, suggesting that Th17-related immunopathology was dominant in the optic nerve. The study concluded that the optic nerve compartment is particularly prone to supporting IL-17-mediated inflammatory immune responses during CNS autoimmunity, and neutralization of IL-17 is sufficient to prevent structural damage to the optic nerve (31). In the present study, the MOG-IgG-seropositive ON patients had a higher concentration of Th17-related cytokines and chemokines than the other types of ON patients. Furthermore, the serum level of IL 17 in the acute phase of the MOG-IgG-seropositive ON was positively correlated with serum MOG-IgG titer, indicating that a higher serum level of IL 17 during the acute phase was related to the MOG-IgG induced. The results of this study have further confirmed that MOG-IgG-related neuroinflammation is immunopathogenetically distinct from classical MS and AQP4-IgG-induced demyelinating disorders. More importantly, these results highlight the important role of the Th17 cells for neuronal demyelination in MOG-IgGinduced neuroinflammation.

There are some limitations in this study. First, the number of $\mathrm{ON}$ patients enrolled in the study was not sufficient, and no follow-up after treatment had been done. Second, we only detected the serum level of the cytokines and chemokines. It would have been much better if we had detected both the CSF and serum levels simultaneously, which could contribute to probing the underlying mechanisms of Th17 cells in MOG-IgG-related demyelinating diseases. Third, we did not compare the serum levels of the cytokines and chemokines of the same patient during the different stages of the disease. Long-term prospective multicenter studies are required to analyze the detailed immunopathologic mechanisms of Th17 cells in different types of demyelinating $\mathrm{ON}$.

In conclusion, this study suggests that Th17 cells were highly activated in MOG-IgG-seropositive ON patients with significantly increased serum cytokines and chemokines. This cytokine/chemokine profiling provides new insights into $\mathrm{ON}$ pathogenesis and is useful in monitoring disease activity. Further research is required to clarify if interference in the Th17 pathway can reduce inflammation in the CNS during disease onset and relapses.

\section{DATA AVAILABILITY STATEMENT}

All datasets presented in this study are included in the article/supplementary material.

\section{ETHICS STATEMENT}

The studies involving human participants were reviewed and approved by Ethics Committee of the Chinese People's Liberation Army General Hospital and Beijing Chaoyang Hospital of Capital Medical University. The patients/participants provided their written informed consent to participate in this study.

\section{AUTHOR CONTRIBUTIONS}

HK, SW, and YT designed and conducted the study. HK, NA, HLiu, and QX collected, managed, analyzed, and interpreted the data. HK and HLi prepared the manuscript. All authors reviewed and made the final approval of the manuscript.

\section{FUNDING}

This work is supported by the National Natural Science Foundation of China (Grant No. 81900849). 


\section{REFERENCES}

1. Toosy AT, Mason DF, Miller DH. Optic neuritis. Lancet Neurol. (2014) 13:83-99. doi: 10.1016/S1474-4422(13)70259-X

2. Jarius S, Wildemann B, Paul F. Neuromyelitis optica: clinical features, immunopathogenesis and treatment. Clin Exp Immunol. (2014) 176:149-64. doi: $10.1111 /$ cei. 12271

3. Jarius S, Wildemann B. AQP4 antibodies in neuromyelitis optica: diagnostic and pathogenetic relevance. Nat Rev Neurol. (2010) 6:383-92. doi: 10.1038/nrneurol.2010.72

4. Jarius S, Ruprecht K, Wildemann B, Kuempfel T, Ringelstein M, Geis C, et al. Contrasting disease patterns in seropositive and seronegative neuromyelitis optica: a multicentre study of 175 patients. J Neuroinflamm. (2012) 9:14. doi: 10.1186/1742-2094-9-14

5. Jarius S, Wildemann B. Aquaporin-4 antibodies (NMO-IgG) as a serological marker of neuromyelitis optica: a critical review of the literature. Brain Pathol. (2013) 23:661-83. doi: 10.1111/bpa.12084

6. Kitley J, Waters P, Woodhall M, Leite MI, Murchison A, George J, et al. Neuromyelitis optica spectrum disorders with aquaporin-4 and myelinoligodendrocyte glycoprotein antibodies: a comparative study. JAMA Neurol. (2014) 71:276-83. doi: 10.1001/jamaneurol.2013.5857

7. Sato DK, Callegaro D, Lana-Peixoto MA, Waters PJ, de Haidar Jorge FM, Takahashi T, et al. Distinction between MOG antibody-positive and AQP4 antibody-positive NMO spectrum disorders. Neurology. (2014) 82:474-81. doi: 10.1212/WNL.0000000000000101

8. Reindl M, Di Pauli F, Rostasy K, Berger T. The spectrum of MOG autoantibody-associated demyelinating diseases. Nat Rev Neurol. (2013) 9:455-61. doi: 10.1038/nrneurol.2013.118

9. Probstel AK, Rudolf G, Dornmair K, Collongues N, Chanson JB, Sanderson NS, et al. Anti-MOG antibodies are present in a subgroup of patients with a neuromyelitis optica phenotype. J Neuroinflamm. (2015) 12:46. doi: 10.1186/s12974-015-0256-1

10. Spadaro M, Gerdes LA, Mayer MC, Ertl-Wagner B, Laurent S, Krumbholz $\mathrm{M}$, et al. Histopathology and clinical course of MOG-antibodyassociated encephalomyelitis. Ann Clin Transl Neurol. (2015) 2:295-301. doi: 10.1002/acn3.164

11. Jarius S, Metz I, Konig FB, Ruprecht K, Reindl M, Paul F, et al. Screening for MOG-IgG and 27 other anti-glial and anti-neuronal autoantibodies in 'pattern II multiple sclerosis' and brain biopsy findings in a MOG-IgG-positive case. Mult Scler. (2016) 22:1541-9. doi: 10.1177/1352458515622986

12. Kothur K, Wienholt L, Tantsis EM, Earl J, Bandodkar S, Prelog K, et al. B cell, Th17, and neutrophil related cerebrospinal fluid cytokine/chemokines are elevated in MOG antibody associated demyelination. PLoS ONE. (2016) 11:e0149411. doi: 10.1371/journal.pone.0149411

13. Ramesh G, MacLean AG, Philipp MT. Cytokines and chemokines at the crossroads of neuroinflammation, neurodegeneration, and neuropathic pain. Mediators Inflamm. (2013) 2013:480739. doi: 10.1155/2013/480739

14. Linhares UC, Schiavoni PB, Barros PO, Kasahara TM, Teixeira B, Ferreira TB, et al. The ex vivo production of IL- 6 and IL- 21 by CD $4+$ T cells is directly associated with neurological disability in neuromyelitis optica patients. J Clin Immunol. (2013) 33:179-89. doi: 10.1007/s10875-012-9780-2

15. Zepp J, Wu L, Li X. IL-17 receptor signaling and T helper 17-mediated autoimmune demyelinating disease. Trends Immunol. (2011) 32:232-9. doi: $10.1016 /$ j.it.2011.02.007

16. The clinical profile of optic neuritis. Experience of the optic neuritis treatment trial. optic neuritis study group. Arch. Ophthalmol. (1991) 109:1673-8. doi: 10.1001/archopht.1991.01080120057025

17. Petzold A, Plant GT. Diagnosis and classification of autoimmune optic neuropathy. Autoimmun Rev. (2014) 13:539-45. doi: 10.1016/j.autrev.2014.01.009

18. Waters PJ, McKeon A, Leite MI, Rajasekharan S, Lennon VA, Villalobos A, et al. Serologic diagnosis of NMO: a multicenter comparison of aquaporin-
4-IgG assays. Neurology. (2012) 78:665-71. doi: 10.1212/WNL.0b013e31 8248dec1

19. Dos Passos GR, Sato DK, Becker J, Fujihara K. Th17 cells pathways in multiple sclerosis and neuromyelitis optica spectrum disorders: pathophysiological and therapeutic implications. Mediators Inflamm. (2016) 2016:5314541. doi: 10.1155/2016/5314541

20. Binger KJ, Corte-Real BF, Kleinewietfeld M. Immunometabolic regulation of interleukin-17-producing $\mathrm{T}$ helper cells: uncoupling new targets for autoimmunity. Front Immunol. (2017) 8:311. doi: 10.3389/fimmu.2017.00311

21. Zhang Y, Yao XY, Gao MC, Ding J, Hong RH, Huang H, et al. Th2 axisrelated cytokines in patients with neuromyelitis optica spectrum disorders. CNS Neurosci Ther. (2018) 24:64-9. doi: 10.1111/cns.12774

22. Huppert J, Closhen D, Croxford A, White R, Kulig P, Pietrowski E, et al. Cellular mechanisms of IL-17-induced blood-brain barrier disruption. FASEB J. (2010) 24:1023-34. doi: 10.1096/fj.09-141978

23. Kebir H, Kreymborg K, Ifergan I, Dodelet-Devillers A, Cayrol R, Bernard M, et al. Human TH17 lymphocytes promote blood-brain barrier disruption and central nervous system inflammation. Nat Med. (2007) 13:1173-5. doi: $10.1038 / \mathrm{nm} 1651$

24. Reboldi A, Coisne C, Baumjohann D, Benvenuto F, Bottinelli D, Lira S, et al. C$\mathrm{C}$ chemokine receptor 6-regulated entry of TH-17 cells into the CNS through the choroid plexus is required for the initiation of EAE. Nat Immunol. (2009) 10:514-23. doi: 10.1038/ni.1716

25. Lindsey JW, Meulmester KM, Brod SA, Nelson F, Wolinsky JS. Variable results after rituximab in neuromyelitis optica. J Neurol Sci. (2012) 317:103-5. doi: 10.1016/j.jns.2012.02.017

26. Varrin-Doyer M, Spencer CM, Schulze-Topphoff U, Nelson PA, Stroud RM, Cree BA, et al. Aquaporin 4-specific T cells in neuromyelitis optica exhibit a Th17 bias and recognize clostridium ABC transporter. Ann Neurol. (2012) 72:53-64. doi: 10.1002/ana.23651

27. Mitsdoerffer M, Kuchroo V, Korn T. Immunology of neuromyelitis optica: a T cell-B cell collaboration. Ann N Y Acad Sci. (2013) 1283:57-66. doi: $10.1111 /$ nyas. 12118

28. Li Y, Wang H, Long Y, Lu Z, Hu X. Increased memory Th17 cells in patients with neuromyelitis optica and multiple sclerosis. J Neuroimmunol. (2011) 234:155-60. doi: 10.1016/j.jneuroim.2011.03.009

29. Jarius S, Ruprecht K, Kleiter I, Borisow N, Asgari N, Pitarokoili K, et al. MOGIgG in NMO and related disorders: a multicenter study of 50 patients. Part 2: Epidemiology, clinical presentation, radiological and laboratory features, treatment responses, and long-term outcome. J Neuroinflamm. (2016) 13:280. doi: 10.1186/s12974-016-0718-0

30. Hoftberger R, Sepulveda M, Armangue T, Blanco Y, Rostasy K, Calvo AC, et al. Antibodies to MOG and AQP4 in adults with neuromyelitis optica and suspected limited forms of the disease. Mult Scler. (2015) 21:866-74. doi: 10.1177/1352458514555785

31. Knier B, Rothhammer V, Heink S, Puk O, Graw J, Hemmer B, et al. Neutralizing IL-17 protects the optic nerve from autoimmune pathology and prevents retinal nerve fiber layer atrophy during experimental autoimmune encephalomyelitis. J. Autoimmun. (2015) 56:34-44. doi: $10.1016 /$ j.jaut.2014.09.003

Conflict of Interest: The authors declare that the research was conducted in the absence of any commercial or financial relationships that could be construed as a potential conflict of interest.

Copyright (C) $2020 \mathrm{Kang}, \mathrm{Li}, \mathrm{Ai}, \mathrm{Liu}, \mathrm{Xu}$, Tao and Wei. This is an open-access article distributed under the terms of the Creative Commons Attribution License (CC BY). The use, distribution or reproduction in other forums is permitted, provided the original author(s) and the copyright owner(s) are credited and that the original publication in this journal is cited, in accordance with accepted academic practice. No use, distribution or reproduction is permitted which does not comply with these terms. 